\title{
Universal Scaling, Beta Function, and Metal-Insulator Transitions
}

\author{
D. N. Sheng and Z. Y. Weng \\ Texas Center for Superconductivity, University of Houston, Houston, Texas 77204-5506
}

(Received 17 February 1999)

\begin{abstract}
We demonstrate a universal scaling form of longitudinal resistance in the quantum critical region of metal-insulator transitions. This is based on numerical results of three-dimensional Anderson transitions (with and without magnetic field), two-dimensional quantum Hall plateau to insulator transition, as well as experimental data of the recently discovered two-dimensional metal-insulator transition. The associated reflection symmetry and a peculiar logarithmic form of the beta function exist over a wide range in which the resistance can change by more than 1 order of magnitude. Interesting implications for the two-dimensional metal-insulator transition are discussed.
\end{abstract}

PACS numbers: 71.30. $+\mathrm{h}, 73.20 . \mathrm{Fz}, 73.20 . J \mathrm{Jc}$

The scaling theory [1] predicted that the noninteracting electrons are always localized in two-dimensional (2D) disordered systems. Recently, a new scaling argument [2] was put forward in order to accommodate the newly found 2D metal-insulator transition (MIT) [3] in zero magnetic field $(B=0)$, where the Coulomb interacthe microscopic mechanism remains unclear [4-6], without violating any general scaling principles the authors assumed the following leading behavior of the "beta function" $\beta(g)=d[\ln (g)] / d[\ln (L)]$ for large conductance $g$ at a finite length scale $L$ :

$$
\beta(g)=(d-2)+A / g^{\alpha}+\ldots,
$$

in which $A$ becomes positive in the aforementioned $B=0$ MIT systems $[3,7,8]$, leading to a metallic phase $(\beta>0)$ at the dimensionality $d=2$.

Since $\beta(g)<0$ at small $g$ (localized region), the beta function is then no longer a monotonic function and has to change sign at some finite $g=g_{c}$, which corresponds to a quantum critical point. Experimental measurements have indicated [3] an exponential form for the conductance with a peculiar reflection symmetry relating the conductance and the resistance on both sides of MIT, which implies [2] the following logarithmic form of the beta function in the quantum critical region (QCR):

$$
\beta(g)=\frac{1}{\nu} \ln \left(g / g_{c}\right) .
$$

In particular, $\nu$ here is the correlation length exponent, and (2) holds at a wide range $\left(1 / 4<g / g_{c}<4\right)$ far beyond a simple small variable expansion around $g=g_{c}$.

The logarithmic form of the beta function (2) looks quite remarkable. Recall that in strong localization limit one may find, exactly, $\beta(g)=\ln (g)-$ const. But the inverse exponent $1 / \nu$ does not show up in front of $\ln (g)$ as in (2), and the corresponding behavior of $g$ should be quite different. So far, there still lacks a good theoretical understanding of (2) from a microscopic model. Nevertheless, one may ask an equally important question: Is (2) a property of the beta function unique for the $B=0$ 2D MIT system or does it actually represent a generic scaling betion presumably becomes very important [3]. Although

havior of quantum phase transitions including other MIT systems with different symmetry and dimensionality? Unfortunately, so far there is no direct answer to this question of how the scaling function of conductance behaves and what the form of the beta function is in the QCR of various MIT systems, although many efforts have been focused on the critical conductance and exponent within each universality class [9-12].

In this paper, we present direct numerical evidence showing that the beta function (2) in fact holds for the following systems as well: three-dimensional (3D) Anderson transitions with and without magnetic field (representing unitary and orthogonal classes, respectively); the 2D electrons in strong magnetic filed, i.e., the quantum Hall effect (QHE) system. Strikingly, $\nu \beta(g)=\ln \left(g / g_{c}\right)$ is found to be a universal function in the QCR where $g / g_{c}$ may change up to 2 orders of magnitude. Correspondingly, the resistance is of an exponential form $\rho_{x x} \propto e^{-s}$ with $s= \pm\left(c_{0} L / \xi\right)^{1 / \nu}$ which also implies a reflection symmetry in the same region [here, $\xi$ is correlation length, and $c_{0}$ is a nonuniversal dimensionless constant $\sim O(1)]$. Thus (2) may well represent a "super" universality property associated with general quantum phase transitions. Furthermore, deep into the metallic region, the beta function shows distinct behavior depending on how the resistance $\rho_{x x}$ deviates from the exponential form: 3D MITs and the $B=02 \mathrm{D}$ MIT experimental data seem to belong to the same group where $d\left[\ln \left(1 / \rho_{x x}\right)\right] / d s$ is a monotonically decreasing function of scaling variable $s$ in the whole scaling region; on the other hand, the QHE system falls into a different group where $d\left[\ln \left(1 / \rho_{x x}\right)\right] / d s$ becomes a monotonically increasing function of $s$. Interestingly, the experimental data of superconductorinsulator transition [13] also fall into the second group, in accord with the speculation [14] that the MIT in the QHE and superconductor-insulator transition may belong to similar universality class.

We consider disordered electron systems described by the Anderson Hamiltonian:

$$
H=-\sum_{\langle i j\rangle} e^{i a_{i j}} c_{i}^{+} c_{j}+\text { H.c. }+\sum_{i} w_{i} c_{i}^{+} c_{i},
$$


where the hopping integral is taken as the unit, and $c_{i}^{+}$ is a fermionic creation operator with $\langle i j\rangle$ referring to the nearest neighboring sites. A uniform magnetic flux per plaquette (along the $z$ direction) can be imposed by requiring $\phi=\sum_{\square} a_{i j}=2 \pi / M$, where the summation runs over four links around a plaquette in the $x-y$ plane. $w_{i}$ is a random potential uniformly distributed between $(-W / 2, W / 2)$.

We first study the 3D electron system without magnetic field $\left(a_{i j}=0\right)$ which belongs to the orthogonal class. The longitudinal conductance $G_{x x}$ is calculated using the Landauer formula [15]. By changing the disorder strength $W$, a metal-insulator transition is found at a critical disorder strength $W_{c}=16.5$ [10] at the Fermi energy $E_{f}=0$, with a critical conductance $G_{c}=0.37$ (in units of $e^{2} / h$ ) and correlation exponent $\nu=1.6$. All the data at different sample sizes $(L=8,10,12,14$, and 16) can be then collapsed onto two branches as a function of $L / \xi$ as shown in Fig. 1(a) ( $\diamond$ curve). Note that the resistance data are plotted in the figure. (More than 2000 configurations are taken in the average for $L=$ 16 , and more for smaller $L$ 's.) A $3 \mathrm{D}$ MIT is similarly obtained in the presence of strong magnetic field (unitary class). We have chosen two different flux strengths $\phi=2 \pi / M: M=5$ at sample sizes $L=10$ and 15 ; and $M=4$ at sample sizes $L=8,12$, and 16 , respectively. All the longitudinal resistance data with different $\phi$ 's and $L$ 's again can be scaled onto two branches $[+$ curve in Fig. 1(a)]. At the critical point, $W_{c}=18.3, \nu=1.43$ [10], and $G_{c}=0.294$ at $E_{f}=0$.

Since the universality of the MIT in the unitary class is distinct from the one of the orthogonal class as expected in the scaling theory $[9,10]$, two scaling curves shown in Fig. 1(a) are generally different from each other. However, if we replot the data in terms of the scaling variable,

$$
s= \pm\left(c_{0} L / \xi\right)^{1 / \nu}
$$

where the sign $+(-)$ corresponds to the metallic (insulating) branch, two curves of longitudinal resistance in the QCR can be precisely scaled together as shown in Fig. 1(b). Here the dimensionless constant $c_{0}$ has the nonuniversal values 2.27 and 1.82 for orthogonal and unitary class, respectively. As shown in the inset of Fig. 1(b), the resistance in the transition region well follows a simple exponential form:

$$
\rho_{x x} / \rho_{c}=\exp (-s)
$$

over a rather broad region: $-2<s<2$ or $1 / 8<$ $\rho_{x x} / \rho_{c}<8$. Because of such a wide range of $s$ (instead of a small parameter expansion), the exponential behavior appears very robust. In the same region, one always finds the so-called reflection symmetry: $\rho_{x x}(s) / \rho_{c}=\rho_{c} / \rho_{x x}(-s)$ between the metallic and insulating branches.

Now let us consider a qualitatively different MIT in the QHE system where 2D electron gas is subjected to a strong magnetic field. By tuning the Fermi energy (or
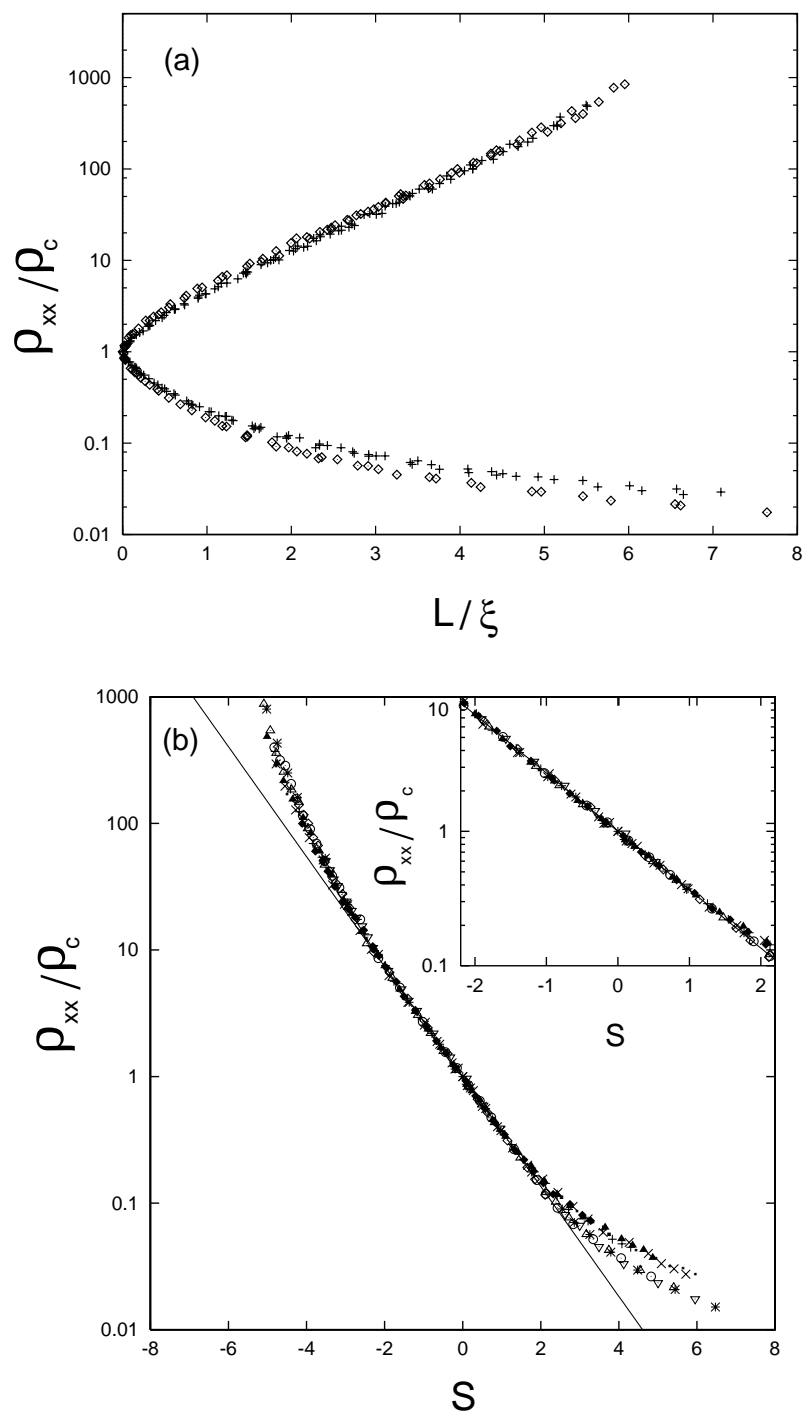

FIG. 1. (a) Finite-size resistance ratio $\rho_{x x} / \rho_{c}$ as a scaling function of $L / \xi$ for 3D Anderson transitions in the absence $(\diamond)$ and presence $(+)$ of magnetic field. (b) $\rho_{x x} / \rho_{c}$ as a function of the scaling variable $s= \pm\left(c_{0} L / \xi\right)^{1 / \nu}$ in the absence $(\diamond, \odot$, $\triangle, \nabla, *$ in increasing $L$ order $)$ and presence $(\bullet,+, \mathbf{\Lambda}, \times, \boldsymbol{\ominus})$ of magnetic field. Inset: The enlarged quantum critical region where $\rho_{x x} / \rho_{c}=e^{-s}$.

the density of electrons) near the lowest Landau level (LL), an insulator to metal transition can be induced which is characterized by a one-parameter scaling theory [11] with an exponent $\nu=2.3$ and $\rho_{c}=1.0$ (in units of $h / e^{2}$ ) [16]. The Hall conductance here is calculated using the Kubo formula. By going to large sample sizes ( $L=24,32,48,56$, and 64), we were able to obtain the scaling behavior of $\rho_{x x}$ for the QHE systems. In Fig. 2, $\rho_{x x}$ is plotted as a function of scaling variable $s$ defined in (3). Again the resistance exhibits the same exponential dependence $\rho_{x x}=\exp (-s)$ (solid line) in the critical region covering a similar wide range of resistance $\left(1 / 5<\rho_{x x} / \rho_{c}<5\right)$ as in the 3D MITs. In Fig. 2, 


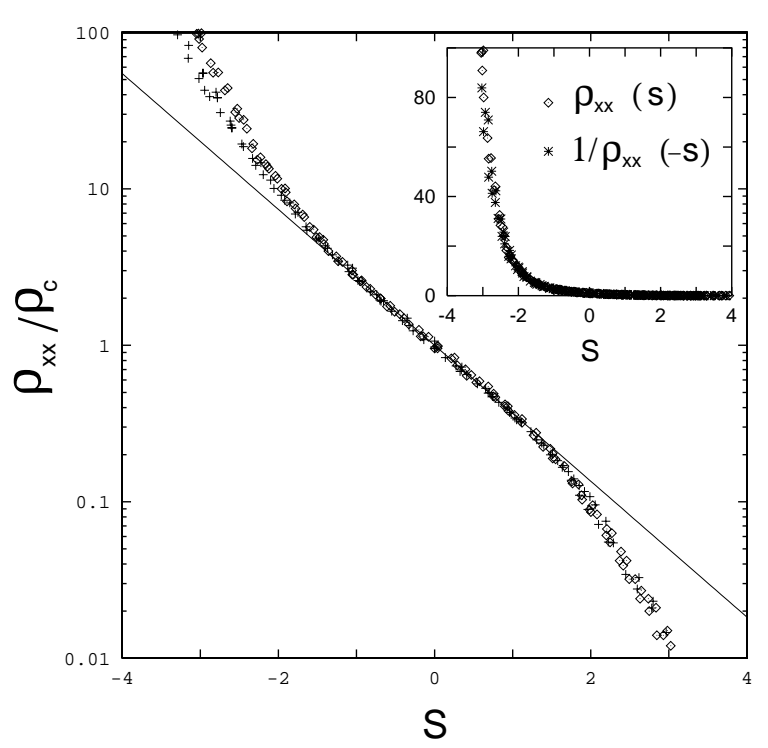

FIG. 2. $\rho_{x x} / \rho_{c}$ as a function of the scaling variable $s$ for the QHE systems both at weak $(\diamond, W=1)$ and strong $(+$, $W=4)$ disorder strengths. Inset: The reflection symmetry of $\rho_{x x}$ at $W=1$.

two different disorder strengths, $W=1$ and $W=4$, are considered which represent weak and strong LL coupling limits, respectively. The corresponding scaling functions start to deviate from the exponential form beyond the critical region and simultaneously become $W$ dependent in the insulating region.

We would like to point out an interesting reflection symmetry for the $W=1$ case: As shown in the inset of Fig. 2, $\rho_{x x}(s)$ on the insulating side and $1 / \rho_{x x}(-s)$ on the metallic side perfectly coincide with each other over the whole scaling region and covering a resistance range $1 / 100<\rho_{x x}<100$ which is way beyond the critical region. Our interpretation is that, at weak disorder $(W=$ $1)$, the particle-hole symmetry is still approximately maintained near the lowest LL such that the Hamiltonian is self-dual $[14,17]$ in the Chern-Simon boson language, which then leads to the wide range of the reflection symmetry. By contrast, when disorder is strong and all the LLs are coupled together without the particle-hole symmetry, the reflection symmetry only exists around the QCR where the exponential behavior (4) is followed.

As demonstrated by the above numerical calculations, the scaling function of longitudinal resistance shows a universal exponential behavior over a wide range in 3D and QHE MITs. In Fig. 3, these data are plotted together with the experimental data obtained in the $B=02 \mathrm{D}$ MIT in the Si sample [3]. Note that the experimental data were measured at finite temperature so the length scale $L$ should be replaced by the dephasing length $L_{\text {in }} \propto$ $T^{-1 / z}$. (Here $z=1$ is the dynamical exponent.) The correlation length $\xi \propto 1 / T_{0} \propto\left|\delta_{n}\right|^{-\nu}$ in the transition region [3] $\left(\delta_{n}\right.$ is the electron density measured from the critical point). So scaling variable $s$ in this case becomes

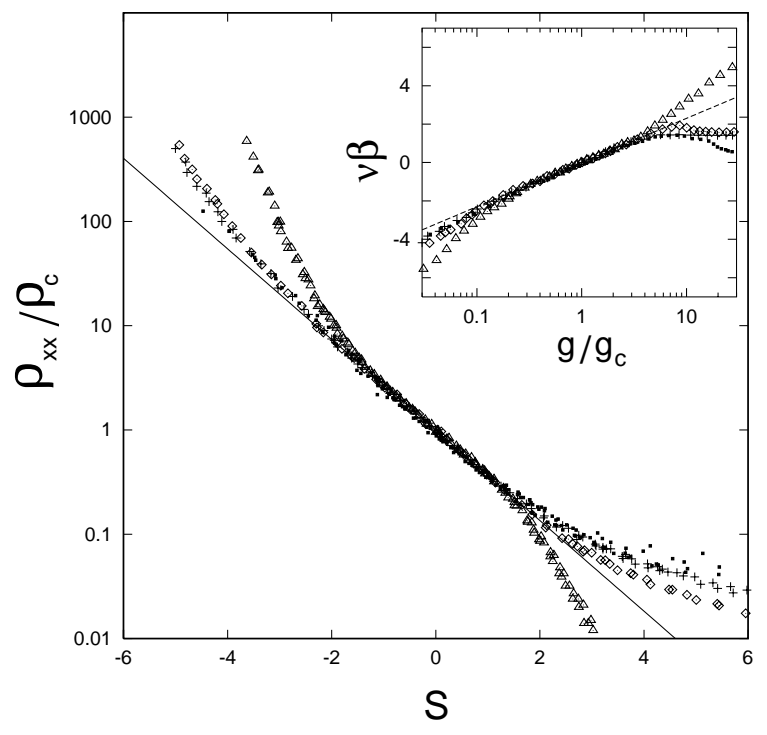

FIG. 3. Experimental data [3] of $B=0$ MIT (O) are plotted together with the resistances calculated for the systems of 3D Anderson transition $(\diamond$ and + ) and the QHE $(\triangle)$. The corresponding $\beta$ functions are shown in the inset which precisely follow the logarithmic form (2) in the QCR.

$\pm\left(c_{0} T_{0} / T\right)^{1 / \nu}$ with $\nu=1.6$ and $c_{0}$ is a dimensionless constant. In Fig. 3, a universal scaling function of the longitudinal resistance is clearly shown for all these systems in the QCR (with $-1.5<s<1.5$ ) despite their different symmetry classes, dimensionalities, and microscopic mechanisms of the MIT. The corresponding beta functions for those systems are shown in the inset of Fig. 3 if we define $g \equiv 1 / \rho_{x x}$, where a straight dashed line represents the logarithmic form of (2) which can be obtained straightforwardly from (4). Note that the beta function is multiplied by the critical exponent $\nu$ in the inset such that the resulting function becomes universal in the QCR. Such a logarithmic form of the beta function for 2D $B=0$ MIT was also obtained (Kim et al. in Ref. [7]) in several other Si samples where the value of $\nu$ can be quite different.

Furthermore, we would like to comment on an interesting trend in the metallic region for those systems. In a weak disorder limit of 3D MITs, the resistance approaches to zero in power law: $\rho_{x x} \sim(\xi / L)=c_{0} s^{-\nu}$. The curve for the $B=02 \mathrm{D}$ MIT system follows very closely to the ones of 3D MITs on the same side of the solid line in Fig. 3 as it drops to zero slower than $\exp (-s)$. In contrast, in the QHE system, the resistance deviates the solid line on the opposite side which means it approaches to zero even quicker than in the QCR. This behavior can be easily seen in its asymptotic form: $\rho_{x x} \sim \sigma_{x x} \sim \exp \left(-s^{\nu} / c_{0}\right)$ at large $s$ limit (since in the QHE plateau region electrons are also localized such that at large $L / \xi$ limit $\sigma_{x x} \sim \exp (-L / \xi)$, $\sigma_{x y}=1$ and $\rho_{x x} \sim \sigma_{x x}$ ). One may then define a generalized dimensionless function as follows:

$$
\beta_{1} \equiv d[\ln (g)] / d s=\nu \beta / s .
$$




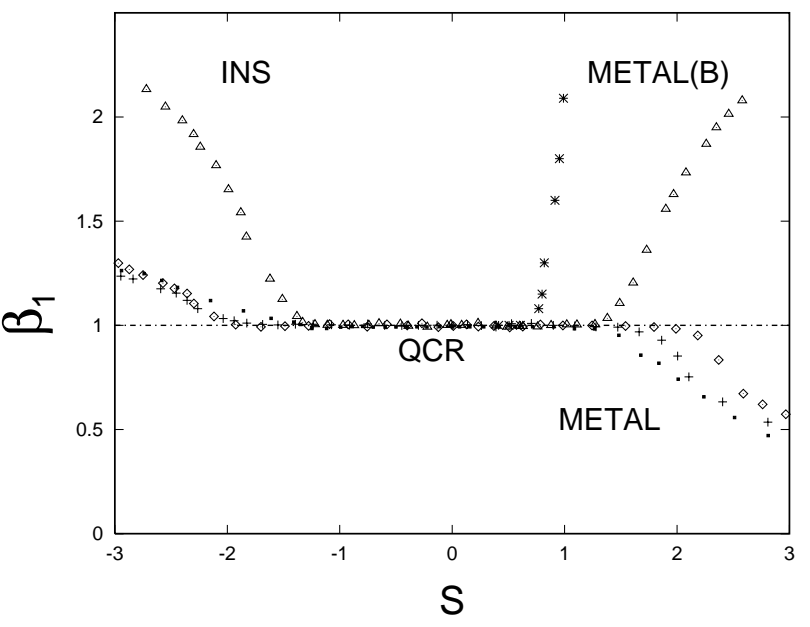

FIG. 4. The function $\beta_{1}$ defined in (5) as a function of the scaling variable $s$ for those systems shown in Fig. 3. In addition, the data for a disorder-tuned superconductorinsulator transition13 in the metallic regime are also shown for comparison $(*)$.

As shown in Fig. 4, all the data fall onto the straight line with $\beta_{1}=1$ in the QCR. In the metallic phase, the distinctive large- $s$ behavior of $\beta_{1}$ separates the metallic regime into two regions. "METAL" denotes the region where $\beta_{1}$ scales to zero which is followed by the 3D MITs as well as the experimental data of the $B=02 \mathrm{D}$ MIT system. On the other hand, $\beta_{1}$ for the MIT in the QHE system diverges to infinity at $s \rightarrow \infty$, which is denoted as the "METAL $(B)$ " region known as the "Bose" metal following the theoretical description [14]. $\beta_{1}$ for disorder-tuned superconductor-insulator transition [13] in the metallic region is also plotted in Fig. 4 (* curve) which indeed shows a quick increase such as in the QHE system. (Here, the scaling variable $s$ is of the same form used in plotting the experimental data of $B=0$ 2D MIT.) According to Ref. [14], these two systems should belong to the same category as classified by the Bose metal here.

We conclude by making several comments on the nature of the $B=02 \mathrm{D}$ MIT systems based on the present work. First, no matter what the microscopic mechanism is, such a 2D MIT seems to belong to a quantum phase transition instead of a classical phase transition (or crossing over): The experimental data of the resistance precisely coincides with those of other known MITs in the QCR, plotted as a function of the scaling variable $\left(L_{\mathrm{in}} / \xi\right)^{1 / \nu}$, which covers a range of the resistance by more than 1 order of magnitude. Second, the reflection symmetry of resistance [3] is the natural consequence of the universal resistance scaling in the QCR. Finally, the metallic phase behaves more like a normal metal than a superconductor as revealed by the classification based on the $\beta_{1}$ function shown in Fig. 4 .
The authors would like to thank S. V. Kravchenko for stimulating discussions and providing his experimental data for comparison. This work is supported by the ARP Grant No. 3652707, and by the State of Texas through Texas Center for Superconductivity at University of Houston.

[1] E. Abrahams, P. W. Anderson, D. C. Licciardello, and T. V. Ramakrishnan, Phys. Rev. Lett. 42, 673 (1979).

[2] V. Dobrosavljevic, E. Abrahams, E. Miranda, and S. Chakravarty, Phys. Rev. Lett. 79, 455 (1997).

[3] S. V. Kravchenko et al., Phys. Rev. B 50, 8039 (1994); 51, 7038 (1995); S. V. Kravchenko et al., Phys. Rev. Lett. 77, 4938 (1996); D. Simonian et al., Phys. Rev. B 55, R13 421 (1997).

[4] S. Chakravarty, L. Yin, and E. Abrahams, Phys. Rev. B 58, R559 (1998); S. Chakravarty et al., cond-mat/ 9805383; C. Castellani et al., Phys. Rev. B 57, R9381 (1998); Q. Si and C. M. Varma, Phys. Rev. Lett. 81, 4951 (1998).

[5] D. Belitz and T.R. Kirkpatrick, Phys. Rev. B 58, 8214 (1998); P. Phillips et al., Nature (London) 395, 253 (1998).

[6] D. N. Sheng and Z. Y. Weng, cond-mat/9901019.

[7] N. Kim et al., cond-mat/9809357; V. M. Pudalov, JETP Lett. 66, 175 (1997); D. Popovic et al., Phys. Rev. Lett. 79, 1543 (1997); D. Simonian et al., Phys. Rev. Lett. 79, 2304 (1997); S. V. Kravchenko et al., Phys. Rev. B 58, 3553 (1998); S. V. Kravchenko et al., cond-mat/9812389.

[8] P. T. Coleridge et al., Phys. Rev. B 56, R12 764 (1997); Y. Hanein et al., Phys. Rev. Lett. 80, 1288 (1998); M. Y. Simons et al., ibid. 80, 1292 (1998); S. J. Papadakis and M. Shayegan, Phys. Rev. B 57, R15 068 (1998); Y. Hanein et al., ibid. 58, R13338 (1998).

[9] F. Wegner, Nucl. Phys. B36, 663 (1989); S. Hikami, Prog. Theor. Phys. Suppl. 107, 213 (1992).

[10] M. Batsch et al., Phys. Rev. Lett. 77, 1552 (1996); K. Slevin and T. Ohtsuki, ibid. 78, 4083 (1997); T. Drose et al., Phys. Rev. B 57, 37 (1998).

[11] B. Huckestein and B. Kramer, Phys. Rev. Lett. 64, 1437 (1990); B. Huckestein, Europhys. Lett. 20, 451 (1992); Y. Huo et al., Phys. Rev. Lett. 70, 481 (1993).

[12] B. Huckestein, Rev. Mod. Phys. 67, 357 (1995); S. Das Sarma, in Perspectives in the Quantum Hall Effect, edited by S. Das Sarma and A. Pinczuk (Wiley, New York, 1997).

[13] Y. Liu et al., Phys. Rev. Lett. 67, 2068 (1991).

[14] M.P. A. Fisher, Phys. Rev. Lett. 65, 923 (1990); M. P. A. Fisher et al., ibid. 64, 587 (1990).

[15] D. S. Fisher and P. A. Lee, Phys. Rev. B 23, 6851 (1981); H. U. Baranger and A. D. Stone, ibid. 40, 8169 (1989).

[16] D. N. Sheng and Z. Y. Weng, Phys. Rev. B 59, R7821 (1999).

[17] D. Shahar et al., Science 274, 589 (1996); P. T. Coleridge and P. Zawadzki, cond-mat/9903246. 